PROCEEDINGS OF THE

AMERICAN MATHEMATICAL SOCIETY

Volume 135, Number 2, February 2007, Pages 437-451

S 0002-9939(06)08621-7

Article electronically published on August 4, 2006

\title{
A REMARK ON LITTLEWOOD-PALEY THEORY FOR THE DISTORTED FOURIER TRANSFORM
}

\author{
W. SCHLAG
}

(Communicated by Andreas Seeger)

\begin{abstract}
We consider the classical theorems of Mikhlin and LittlewoodPaley from Fourier analysis in the context of the distorted Fourier transform. The latter is defined as the analogue of the usual Fourier transform as that transformation which diagonalizes a Schrödinger operator $-\Delta+V$. We show that for such operators which display a zero energy resonance the full range $1<p<\infty$ in the Mikhlin theorem cannot be obtained: in the radial, threedimensional case it shrinks to $\frac{3}{2}<p<3$.
\end{abstract}

\section{INTRODUCTION}

The fundamental Littlewood-Paley theorem states that for any choice of $1<p<$ $\infty$ there is a constant $C_{p, d}$ such that

$$
C_{p, d}^{-1}\|f\|_{p} \lesssim\|S f\|_{p} \lesssim C_{p, d}\|f\|_{p}
$$

for all Schwartz functions $f \in \mathcal{S}\left(\mathbb{R}^{d}\right)$; see Stein [Ste1], Ste2]. Here

$$
S f:=\left(\sum_{j=-\infty}^{\infty}\left|\Delta_{j} f\right|^{2}\right)^{\frac{1}{2}}
$$

is the Littlewood-Paley square function which is associated with a dyadic partition of unity

$$
\sum_{j=-\infty}^{\infty} \hat{\psi}\left(2^{-j} \xi\right)=1 \quad \forall \xi \neq 0,
$$

and $\Delta_{j} f=\left(\hat{\psi}\left(2^{-j} \cdot\right) \hat{f}\right)^{\vee}$. Closely related is the Mikhlin multiplier theorem, which states that for all $1<p<\infty$

$$
\left\|(\mu \hat{f})^{\vee}\right\|_{p} \leq C_{p, d}\|f\|_{p}
$$

provided $\mu \in C^{d+2}\left(\mathbb{R}^{d} \backslash\{0\}\right)$ satisfies the derivative bounds

$$
\left|\partial^{\alpha} \mu(\xi)\right| \leq C_{\alpha}|\xi|^{-|\alpha|}
$$

for all $0 \leq|\alpha| \leq d+2$, say (less is needed). The point here is that the kernel $\hat{\mu}$ is a Calderon-Zygmund kernel. In particular, it decays like $|x|^{-d}$ at infinity (and in

Received by the editors August 29, 2005.

2000 Mathematics Subject Classification. Primary 35J10, 42B15; Secondary 35P10, 42B25.

Key words and phrases. Littlewood-Paley theory, distorted Fourier transform, zero energy resonances of Schrödinger operators.

The author was partially supported by NSF grant DMS-0300081 and a Sloan Fellowship.

(C)2006 American Mathematical Society Reverts to public domain 28 years from publication 
general no better, which means that $p=1$ or $p=\infty$ are forbidden). To pass from the multiplier theorem to the Littlewood-Paley square function bound one checks that the random function

$$
\mu(\xi):=\sum_{j} \pm \hat{\psi}\left(2^{-j} \xi\right)
$$

where \pm are i.i.d. symmetric Bernoulli, are Mikhlin multipliers uniformly in the choice of the signs \pm . The bound (11) is then obtained by combining (2) with the Khintchin inequality.

To purpose of this note is to investigate to what extent these same bounds also hold relative to the distorted Fourier transform (we will restrict ourselves to $d=3$ ). The latter here refers to a basis of functions which diagonalize the Schrödinger operator $H=-\Delta+V$ on its continuous spectral subspace. More precisely, we need to assume that the real-valued potential $V$ decays sufficiently rapidly to guarantee that this distorted Fourier basis for $H$ exists. This basis, which we denote by $\{e(x, \xi)\}_{\xi \in \mathbb{R}^{d}}$, consists of solutions of the Lippman-Schwinger equation

$$
e(\cdot, \xi)=e_{\xi}-R_{V}^{-}\left(\xi^{2}\right) V e_{\xi}, \quad e_{\xi}(x)=e^{i x \cdot \xi} .
$$

See Agmon $\mathrm{Agm}$ for conditions that guarantee the solvability of this equation as well as for the Fourier expansion and inversion theorem in this setting 1 Denote the distorted Fourier transform by

$$
\mathcal{F}_{V} f(\xi):=(2 \pi)^{-\frac{3}{2}} \int_{\mathbb{R}^{3}} \overline{e(x, \xi)} f(x) d x,
$$

and, for any bounded $\mu=\mu(\xi)$ define a multiplier operator by

$$
\mathcal{M}_{\mu} f=\mathcal{F}_{V}^{-1}\left(\mu \mathcal{F}_{V} f\right) .
$$

Let $\sum_{j} \psi\left(2^{-j} \xi\right)=1$ be the usual Littlewood-Paley decomposition of unity and define a square function

$$
S_{H} f(x)=\left(\sum_{j}\left|\psi\left(2^{-j} \sqrt{H}\right) f\right|^{2}(x)\right)^{\frac{1}{2}}
$$

on the continuous subspace $L_{c}^{2}\left(\mathbb{R}^{3}\right)$ relative to $H$. It is of course natural to develop analogues of the classical Mikhlin and Littlewood-Paley theorems in this perturbed setting. By the fundamental work of Yajima Yaj the wave operators are bounded on $L^{p}\left(\mathbb{R}^{3}\right), 1 \leq p \leq \infty$, provided $V$ decays faster than a fifth power and $H$ has neither an eigenvalue nor a resonance at zero energy. The latter here refers to the fact that there are no solutions of the equation $H f=0, f \in L^{2,-\sigma}\left(\mathbb{R}^{3}\right)$ $:=\langle x\rangle^{\sigma} L^{2}\left(\mathbb{R}^{3}\right), \sigma>\frac{1}{2}$. Equivalently, it means that the perturbed resolvents $(-\Delta+V-z)^{-1}$ remain bounded as operators from $L^{2, \sigma} \rightarrow L^{2,-\sigma}$ with $\sigma>1$ as $z \rightarrow 0$ in the upper half-plane $\operatorname{Im} z>0$. Since the wave operators intertwine $-\Delta$ and $H$ restricted to its continuous subspace, we conclude from Yajima's work that (11) and (2) remain valid for $S_{H}$ and $\mathcal{M}_{\mu}$ provided we are on the continuous subspace $L_{c}^{2}\left(\mathbb{R}^{3}\right)$.

In some applications, however, operators $H$ arise that do exhibit resonances and/or eigenfunctions at zero energy. One such example, which served as motivation

\footnotetext{
${ }^{1}$ We are not after rough or slowly decaying potentials here. In fact, we will consider only bounded potentials that decay like $|x|^{-3-\varepsilon}$.
} 
for this work, was encountered by Krieger and the author in KriSch: As discovered by Aubin [Aub] and Talenti [Tal], the functions

$$
\phi(x, a)=(3 a)^{\frac{1}{4}}\left(1+a|x|^{2}\right)^{-\frac{1}{2}}
$$

defined in $\mathbb{R}^{3}$ for each $a>0$ solve

$$
-\Delta \phi(\cdot, a)-\phi^{5}(\cdot, a)=0 .
$$

Indeed, these functions are the unique extremizers (up to translations) of the critical Sobolev imbedding $\dot{H}^{1}\left(\mathbb{R}^{3}\right) \rightarrow L^{6}\left(\mathbb{R}^{3}\right)$, and (7) is the associated Euler-Lagrange equation. Hence,

$$
H_{a}:=-\Delta-5 \phi^{4}(\cdot, a)
$$

satisfies

$$
H_{a} \partial_{a} \phi(\cdot, a)=0, \quad H_{a} \nabla \phi(\cdot, a)=0 .
$$

Since $\left|\partial_{a} \phi(x, a)\right| \asymp|x|^{-1}$ as $|x| \rightarrow \infty$, it follows that $\partial_{a} \phi \in L^{2,-\frac{1}{2}-\varepsilon} \backslash L^{2}\left(\mathbb{R}^{3}\right)$. This means that $H_{a}$ has both a resonance and an eigenvalue at zero energy. In KriSch, the functions $\phi(\cdot, a)$ were considered as static solutions of the wave equation $\square \psi-$ $\psi^{5}=0$. They are linearly unstable, but they admit stable manifolds of codimension one, at least for radial data; see [KriSch]. In order to show this, the following linear bound was used (amongst others): Fix $a>0$ and restrict $H_{a}$ to the radial subspace $L_{\text {rad }}^{2}\left(\mathbb{R}^{3}\right)$. Then there exists $c_{0} \neq 0$ so that

$$
\frac{\sin \left(t \sqrt{H_{a}}\right)}{\sqrt{H_{a}}}=c_{0} \partial_{a} \phi(\cdot, a) \otimes \partial_{a} \phi(\cdot, a)+\mathcal{S}_{a}(t),
$$

where $\mathcal{S}_{a}(t)$ is dispersive in the following sense:

$$
\left\|\mathcal{S}_{a}(t) f\right\|_{\infty} \lesssim t^{-1}\|f\|_{W^{1,1}\left(\mathbb{R}^{3}\right)} .
$$

However, Strichartz estimates are still lacking for $\mathcal{S}_{a}(t)$.

What we develop here is a Littlewood-Paley theorem for $H_{a}$ (or more general operators with radial potentials) restricted to the subspace of radial functions. It turns out that due to the singularity of the resolvent at zero energy which results from the resonance, the Mikhlin multiplier theorem can only hold in the range $\frac{3}{2}<p<3$. In this range, we show that it does hold at least for radial functions. The reason for this smaller range of $p$ lies with the decay of the kernel associated with $\mathcal{M}_{\mu}$. It decays at infinity like $|x|^{-2}$ rather than $|x|^{-3}$, as in the Calderon-Zygmund case. This forces $p>\frac{3}{2}$ and by duality also $p<3$. Hence, we can only prove the Littlewood-Paley theorem in this same range of $p$ (and for radial functions). This is inadequate for deriving Strichartz estimates for $\mathcal{S}_{a}(t)$. Consequently, it seems reasonable to believe that the latter requires a Littlewood-Paley theory which is based on the regular part of the spectral measure of $H_{a}$, just as much as $\mathcal{S}_{a}(t)$ is the sine evolution of the regular part of the spectral measure. However, we do not pursue that here.

A very detailed analysis of Besov spaces relative to the distorted Fourier transform was carried out by Jensen and Nakamura [JenNak1], JenNak2]. However, these authors only consider inhomogeneous Besov spaces. In other words, they carry out the dyadic partition only for large energies, and small energies are treated as a single block. This is not only quite different from the full square function, but is also insufficient for proving Strichartz estimates for the operator $\mathcal{S}_{a}(t)$. 


\section{LitTleWOOD-PALEY THEORY IN THE PERTURBED RADIAL CASE}

Let $H=-\Delta+V$ with a real-valued, radial, decaying potential $V$ in $\mathbb{R}^{3}$ and with no eigenvalue, but a resonance at zero 2 As for the decay, we shall assume that $|V(r)| \lesssim\langle r\rangle^{-3-\varepsilon}$ with $\varepsilon>0$. It will also be convenient to assume that $\left|V^{\prime}(r)\right| \lesssim\langle r\rangle^{-4-\varepsilon}$ with $\varepsilon>0$. In this section, we shall only consider radial multipliers operating on radial functions. Let $\{e(x, \xi)\}$ be the distorted Fourier basis for $H$; see above. For any $r, k>0$ and $x$ with $|x|=r$, let

$$
\tilde{e}(r, k)=\frac{r k}{4 \pi} \int_{S^{2}} e(x, k \omega) \sigma(d \omega),
$$

which is well defined by the radial symmetry of $V$. Then, for any $k>0$,

$$
\left(-\partial_{r r}+V\right) \tilde{e}(r, k)=k^{2} \tilde{e}(r, k) \quad \forall r>0, \quad \tilde{e}(0, k)=0,
$$

and, moreover, for any radial Schwartz functions $f, g$

$$
\begin{aligned}
\left\langle\mathcal{M}_{\mu} f, g\right\rangle=\left\langle\mathcal{F}_{V}^{-1}\left(\mu \mathcal{F}_{V} f\right), g\right\rangle & \\
= & (2 \pi)^{-3} \int_{0}^{\infty} \int_{0}^{\infty} \int_{0}^{\infty} \mu(k) \int_{S^{2}} \int_{S^{2}} \int_{S^{2}} e\left(r_{1} \omega_{1}, k \omega\right) \overline{e\left(r_{2} \omega_{2}, k \omega\right)} \sigma\left(d \omega_{1}\right) \sigma\left(d \omega_{2}\right) \sigma(d \omega) \\
& \times k^{2} d k r_{1}^{2} f\left(r_{1}\right) r_{2}^{2} g\left(r_{2}\right) d r_{1} d r_{2} \\
& =\frac{2}{\pi} \int_{0}^{\infty} \int_{0}^{\infty} \int_{0}^{\infty} \int_{0}^{\infty} \mu(k) \tilde{e}\left(r_{1}, k\right) \overline{\tilde{e}\left(r_{2}, k\right)} d k r_{1} f\left(r_{1}\right) r_{2} g\left(r_{2}\right) d r_{1} d r_{2} \\
(11) \quad & \left.\left\langle\tilde{\mathcal{F}}_{V}^{-1}\left(\mu \tilde{\mathcal{F}}_{V} \tilde{f}\right), \tilde{g}\right\rangle=:\left\langle r_{1}, k\right) \overline{\tilde{e}\left(r_{2}, k\right)} d k \tilde{f}\left(r_{1}\right) \tilde{g}\left(r_{2}\right) d r_{1} d r_{2} \tilde{f}, \tilde{g}\right\rangle,
\end{aligned}
$$

where $\tilde{f}=\sqrt{4 \pi} r f(r), \tilde{g}=\sqrt{4 \pi} r g(r)$ and 3

$$
\left(\tilde{\mathcal{F}}_{V} \tilde{f}\right)(k)=\sqrt{\frac{2}{\pi}} \int_{0}^{\infty} \overline{\tilde{e}(\rho, k)} \tilde{f}(\rho) d \rho .
$$

This shows that it suffices to study spectral multipliers associated with the operator on $L^{2}(0, \infty)$ given by

$$
\tilde{H}:=-\partial_{r r}+V(r)
$$

with a Dirichlet condition at $r=0$. Our normalizations are chosen so that $f \mapsto \tilde{f}$ as a map $L_{\text {rad }}^{2}\left(\mathbb{R}^{3}\right) \rightarrow L^{2}(0, \infty)$ is unitary, and so that both $\mathcal{F}_{V}$ and $\tilde{\mathcal{F}}_{V}$ are unitary on their respective $L_{c}^{2}$ spaces. In the free case of course $e(x, \xi)=e^{i x \cdot \xi}$ and $\tilde{e}(r, k)=$ $\sin (r k)$.

Our first goal in this section is to prove the following result. Note that we do not make any assumption on eigenvalues or resonances at zero energy.

\footnotetext{
${ }^{2}$ Hence, the results from this section apply to $H_{a}$ as in (7), provided we restrict $H_{a}$ to $L_{\mathrm{rad}}^{2}\left(\mathbb{R}^{3}\right)$.

${ }^{3}$ For a particularly simple derivation of the Fourier expansion theorem in the half-line case, see Gesztesy and Zinchenko [GesZin].
} 
Proposition 1. Let $H=-\Delta+V(r)$ with $|V(r)| \lesssim\langle r\rangle^{-\beta}$ and $\left|V^{\prime}(r)\right| \lesssim\langle r\rangle^{-\beta-1}$ for some $\beta>3$. Let $\mu$ be an arbitrary radial function in $C^{3}\left(\mathbb{R}^{3} \backslash\{0\}\right)$ satisfying 4 $\left|\mu^{(\ell)}(k)\right| \leq k^{-\ell}$ for all $k>0$ and $\ell=0,1,2,3$. Then $\mathcal{F}_{V}^{-1}\left(\mu \mathcal{F}_{V} f\right)=: \mathcal{M}_{\mu} f$ satisfies the bounds

$$
\left\|\mathcal{M}_{\mu} f\right\|_{p} \leq C(V, p)\|f\|_{p}
$$

for all $3 / 2<p<3$ and all radial $f \in L^{p}\left(\mathbb{R}^{3}\right)$.

The proof of this proposition exploits the reduction to the half-line operator $\widetilde{\mathcal{M}}_{\mu}$ from (11). It will be based on the theory of $A_{p}$ weights on the line; see Stein Ste2, Chapter V. The main estimates will be given in two separate pieces for large and small $k$; see Lemma 2 and 3 below, respectively. We need to develop some standard background material before embarking on the proofs.

The solution to (10) is unique up to normalization. We remark that in our application (10) has a bounded, nonzero solution for $k=0$ due to the resonance at zero. For arbitrary $k>0$,

$$
\tilde{e}(r, k)=c_{+}(k) f(r, k)+c_{-}(k) \overline{f(r, k)},
$$

where $f(r, k)$ are the Jost functions, i.e.,

$$
\left(-\partial_{r r}+V\right) f(r, k)=k^{2} f(r, k) \quad \forall r>0, \quad f(r, k) \sim e^{i r k} \text { as } r \rightarrow \infty .
$$

They are the unique solutions of the Volterra equation

$$
f(r, k)=e^{i r k}+\int_{r}^{\infty} \frac{\sin \left(k\left(r^{\prime}-r\right)\right)}{k} V\left(r^{\prime}\right) f\left(r^{\prime}, k\right) d r^{\prime} \quad \forall r \geq 0 .
$$

If $k=0$, then

$$
f(r, 0)=1+\int_{r}^{\infty}\left(r^{\prime}-r\right) V\left(r^{\prime}\right) f\left(r^{\prime}, 0\right) d r^{\prime} \quad \forall r \geq 0 .
$$

It is standard that

$$
\sup _{r, k \geq 0}|f(r, k)| \leq \exp \left(\int_{0}^{\infty} r^{\prime}\left|V\left(r^{\prime}\right)\right| d r^{\prime}\right)<\infty .
$$

The resolvent of $-\partial_{r r}$ on $L^{2}(0, \infty)$ with Dirichlet boundary condition is

$$
R_{0}^{+}\left(k^{2}\right)\left(r, r^{\prime}\right)=k^{-1} \sin (r k) e^{i r^{\prime} k} \chi_{\left[0<r<r^{\prime}\right]}+k^{-1} \sin \left(r^{\prime} k\right) e^{i r k} \chi_{\left[0<r^{\prime}<r\right]} .
$$

Therefore, by the Lippman-Schwinger equation for $\tilde{H}$,

$$
\begin{aligned}
\tilde{e}(r, k)= & \sin (r k)-R_{0}^{+}\left(k^{2}\right) V \tilde{e}(\cdot, k)(r) \\
= & \sin (r k)-k^{-1} \int_{0}^{r} \sin \left(r^{\prime} k\right) e^{i r k} V\left(r^{\prime}\right) \tilde{e}\left(r^{\prime}, k\right) d r^{\prime} \\
& -k^{-1} \int_{r}^{\infty} \sin (r k) e^{i r^{\prime} k} V\left(r^{\prime}\right) \tilde{e}\left(r^{\prime}, k\right) d r^{\prime},
\end{aligned}
$$

\footnotetext{
${ }^{4}$ Less is needed on $\mu$, but we ignore these refinements.
} 
which implies that

$$
\begin{aligned}
\partial_{r} \tilde{e}(0, k) & =k-\int_{0}^{\infty} e^{i r^{\prime} k} V\left(r^{\prime}\right) \tilde{e}\left(r^{\prime}, k\right) d r^{\prime} \\
& =k-c_{+}(k) \int_{0}^{\infty} e^{i r^{\prime} k} V\left(r^{\prime}\right) f\left(r^{\prime}, k\right) d r^{\prime}-c_{-}(k) \int_{0}^{\infty} e^{i r^{\prime} k} V\left(r^{\prime}\right) \overline{f\left(r^{\prime}, k\right)} d r^{\prime} .
\end{aligned}
$$

Hence $c_{+}(k), c_{-}(k)$ solve the system

$$
\begin{array}{r}
c_{+}(k) f(0, k)+c_{-}(k) \overline{f(0, k)}=0, \\
c_{+}(k)\left(\partial_{r} f(0, k)+\int_{0}^{\infty} e^{i r^{\prime} k} V\left(r^{\prime}\right) f\left(r^{\prime}, k\right) d r^{\prime}\right) \\
+c_{-}(k)\left(\overline{\partial_{r} f(0, k)}+\int_{0}^{\infty} e^{i r^{\prime} k} V\left(r^{\prime}\right) \overline{f\left(r^{\prime}, k\right)} d r^{\prime}\right)=k .
\end{array}
$$

Let $D(k)$ denote the determinant of this system. Then

$$
\begin{aligned}
D(k)= & f(0, k) \overline{\partial_{r} f(0, k)}-\overline{f(0, k)} \partial_{r} f(0, k) \\
& +\int_{0}^{\infty} e^{i r^{\prime} k} V\left(r^{\prime}\right)\left[f(0, k) \overline{f\left(r^{\prime}, k\right)}-\overline{f(0, k)} f\left(r^{\prime}, k\right)\right] d r^{\prime} .
\end{aligned}
$$

Since

$$
\begin{array}{r}
f(0, k)=1+k^{-1} \int_{0}^{\infty} \sin \left(r^{\prime} k\right) V\left(r^{\prime}\right) f\left(r^{\prime}, k\right) d r^{\prime}, \\
f^{\prime}(0, k)=\partial_{r} f(0, k)=i k-\int_{0}^{\infty} \cos \left(r^{\prime} k\right) V\left(r^{\prime}\right) f\left(r^{\prime}, k\right) d r^{\prime},
\end{array}
$$

we further conclude that

$$
\begin{aligned}
D(k) & =-2 i k \operatorname{Re}(f(0, k))+i \int_{0}^{\infty} \sin \left(r^{\prime} k\right) V\left(r^{\prime}\right) 2 i \operatorname{Im}\left[f(0, k) \overline{f\left(r^{\prime}, k\right)}\right] d r^{\prime} \\
& =-2 i k \operatorname{Re}(f(0, k))+2 k \operatorname{Im}[f(0, k)(1-\overline{f(0, k)})]=-2 i k f(0, k) .
\end{aligned}
$$

Evaluating the Wronskian $W[f(\cdot, k), \overline{f(\cdot, k)}]$ at $r=0$ and $r=\infty$ implies that

$$
\operatorname{Im}\left(f(0, k) \overline{f^{\prime}(0, k)}\right)=-k,
$$

and thus

$$
C(V)>|f(0, k)|>C(V)^{-1} k(1+k)^{-1} .
$$

Finally, for all $k \neq 0$

$$
c_{+}(k)=\frac{1}{2 i} \frac{\overline{f(0, k)}}{f(0, k)}, \quad c_{-}(k)=-\frac{1}{2 i},
$$


and thus

$$
\tilde{e}(r, k)=\frac{f(r, k) \overline{f(0, k)}-\overline{f(r, k)} f(0, k)}{2 i f(0, k)} .
$$

Since $f(r, k)=e^{i r k}+O\left(k^{-1}\right)$, it follows that

$$
\tilde{e}(r, k)=\sin (r k)+O\left(k^{-1}\right) \text { as } k \rightarrow \infty .
$$

The behavior at $k=0$ depends on whether or not there is a nonzero bounded solution $y$ to $\tilde{H} y=0, y(0)=0$. It is easy to see that this dichotomy is equivalent with the dichotomy $f(0,0)=0$ vs. $f(0,0) \neq 0$. Indeed, since $|V(x)| \lesssim\langle x\rangle^{-3-\varepsilon}$, all such solutions $y$ are given by

$$
y(r)=c_{1}+c_{2} r+\int_{r}^{\infty}\left(r^{\prime}-r\right) V\left(r^{\prime}\right) y\left(r^{\prime}\right) d r^{\prime} .
$$

Hence, $y \in L^{\infty}(0, \infty)$ iff $c_{2}=0$ in which case and $y(r)=c_{1} f(r, 0)$. Hence, there exists a nonzero bounded solution $y$ to $\tilde{H} y=0, y(0)=0$ iff $f(0,0)=0$. In our application (7), $H_{a}\left(\partial_{a} \phi(\cdot, a)\right)=0$ and thus $\tilde{H}_{a}\left(r \partial_{a} \phi(\cdot, a)\right)=0$ with a bounded solution $y(r)=r \partial_{a}(r, a)$ vanishing at $r=0$. Therefore, $f(0,0)=0$, which implies that (16) is optimal in that case.

In what follows, we will assume that $f(0,0)=0$. This is the harder case, and $f(0,0) \neq 0$ is also implicit in what we are doing below. Hence, differentiating (15) in $k$ then proves that

$$
\operatorname{Im}\left(\partial_{k} f(0,0) \overline{f^{\prime}(0,0)}\right)=-1, \quad \partial_{k} f(0,0) \neq 0,
$$

and by d'Hospital's rule $c_{+}(k)$ is continuous on $[0, \infty)$ with

$$
c_{+}(0)=\frac{1}{2 i} \frac{\overline{\partial_{k} f(0,0)}}{\partial_{k} f(0,0)} .
$$

This requires that $\langle r\rangle^{2} V \in L^{1}$, which is the case here. However, we cannot guarantee that $c_{+} \in C^{1}[0, \infty)$, since that would require $\langle r\rangle^{3} V \in L^{1}$. Nevertheless, in view of (14) one has $f(0, k) \in C^{\infty}(0, \infty)$ and thus also $c_{+} \in C^{\infty}(0, \infty)$.

The following lemma reduces $\widetilde{\mathcal{M}}_{\mu}$ to manageable pieces in the high-energy case.

Lemma 2. Let $\mu \in C^{2}(0, \infty)$ with $\left|\mu^{(\ell)}(k)\right| \leq k^{-\ell}$ for all $k>0$ and $\ell=0,1,2$. Assume further that $\mu(k)=0$ for all $0<k<1$. Then the kernel $K\left(r, r^{\prime}\right)$ of $\widetilde{\mathcal{M}}_{\mu}=\tilde{\mathcal{F}}_{V}^{-1} \mu \tilde{\mathcal{F}}_{V}$ satisfies

$$
K=K_{1}+\widetilde{K}_{1}+K_{2}+K_{3}
$$

where, with $m_{0}(r)=-\int_{r}^{\infty} V\left(r^{\prime}\right) d r^{\prime}$,

$$
\begin{aligned}
K_{1}\left(r, r^{\prime}\right) & =\frac{1}{2} \int_{0}^{\infty} \cos \left(\left(r-r^{\prime}\right) k\right) \mu(k) d k \\
\widetilde{K}_{1}\left(r, r^{\prime}\right) & =\frac{1}{4} \int_{0}^{\infty} \sin \left(\left(r-r^{\prime}\right) k\right) \frac{\mu(k)}{k} d k \cdot\left(m_{0}(r)-m_{0}\left(r^{\prime}\right)\right), \\
\left|K_{2}\left(r, r^{\prime}\right)\right| & \lesssim\left\langle r-r^{\prime}\right\rangle^{-2}, \\
\left|K_{3}\left(r, r^{\prime}\right)\right| & \lesssim\left(r+r^{\prime}\right)^{-1}
\end{aligned}
$$

for all $r, r^{\prime} \in(0, \infty)$. In particular, all these kernels are $L^{p}(0, \infty)$-bounded for $1<p<\infty$. 
Proof. It will be convenient to assume that $\mu(k)=0$ for large values of $k$, but the bounds will not depend on this additional cut-off so that it can be removed in the end. The kernel of $\mathcal{M}_{\mu}$ is

$$
\begin{aligned}
K\left(r, r^{\prime}\right)= & \int_{0}^{\infty} \mu(k) \tilde{e}(r, k) \overline{\tilde{e}\left(r^{\prime}, k\right)} d k \\
= & \int_{0}^{\infty} \mu(k)\left(c_{+}(k) f(r, k)+c_{-}(k) \overline{f(r, k)}\right)\left(\overline{c_{+}(k)} \overline{f\left(r^{\prime}, k\right)}+\overline{c_{-}(k)} f\left(r^{\prime}, k\right)\right) d k \\
(18) \quad= & \frac{1}{4} \int_{0}^{\infty} e^{i\left(r-r^{\prime}\right) k} \mu(k) m(r, k) \overline{m\left(r^{\prime}, k\right)} d k \\
& +\frac{1}{4} \int_{0}^{\infty} e^{-i\left(r-r^{\prime}\right) k} \mu(k) m\left(r^{\prime}, k\right) \overline{m(r, k)} d k \\
& +\frac{1}{2 i} \int_{0}^{\infty} e^{i\left(r+r^{\prime}\right) k} \mu(k) c_{+}(k) m(r, k) m\left(r^{\prime}, k\right) d k \\
- & \frac{1}{2 i} \int_{0}^{\infty} e^{-i\left(r+r^{\prime}\right) k} \mu(k) \overline{c_{+}(k)} \overline{m(r, k) m\left(r^{\prime}, k\right)} d k \\
= & K^{(+,+)}\left(r, r^{\prime}\right)+K^{(-,-)}\left(r, r^{\prime}\right)+K^{(+,-)}\left(r, r^{\prime}\right)+K^{(-,+)}\left(r, r^{\prime}\right),
\end{aligned}
$$

where we have set $f(r, k)=e^{i r k} m(r, k)$. Thus,

$$
m(r, k)=1+\int_{r}^{\infty} \frac{e^{2 i k\left(r^{\prime}-r\right)}-1}{2 i k} V\left(r^{\prime}\right) m\left(r^{\prime}, k\right) d r^{\prime},
$$

which shows that

$$
\sup _{r>0}|m(r, k)-1| \lesssim k^{-1}, \quad \sup _{r, k>0}\left|\partial_{r} m(r, k)\right|<\infty .
$$

Using a derivative of $V$, we can improve on the first bound by integrating by parts in (20). Indeed, one checks that

$$
m(r, k)=1+\frac{1}{2 i k} m_{0}(r)+\frac{1}{k^{2}} m_{1}(r, k),
$$

where $m_{1}$ satisfies

$$
\sup _{r>0}\left|\partial_{k}^{j} m_{1}(r, k)\right| \lesssim 1, \quad j=0,1,2
$$

Therefore, also

$$
m(r, k) \overline{m\left(r^{\prime}, k\right)}=1+\frac{1}{2 i k}\left(m_{0}(r)-m_{0}\left(r^{\prime}\right)\right)+\frac{1}{k^{2}} \tilde{m}_{1}\left(r, r^{\prime}, k\right),
$$


where $\tilde{m}_{1}$ satisfies (21) uniformly in $r, r^{\prime}$. This implies that

$$
\begin{aligned}
& \left|K^{(+,+)}\left(r, r^{\prime}\right)-\frac{1}{4} \int_{0}^{\infty} e^{i\left(r-r^{\prime}\right) k} \mu(k) d k-\frac{1}{8 i k}\left(m_{0}(r)-m_{0}\left(r^{\prime}\right)\right) \int_{0}^{\infty} e^{i\left(r-r^{\prime}\right) k} \frac{\mu(k)}{k} d k\right| \\
& \quad \lesssim\left\langle r-r^{\prime}\right\rangle^{-2} .
\end{aligned}
$$

Since it is standard that

$$
\int_{0}^{\infty} e^{i\left(r-r^{\prime}\right) k} \mu(k) d k \text { and } \quad \int_{0}^{\infty} e^{i\left(r-r^{\prime}\right) k} \frac{\mu(k)}{k} d k
$$

are singular integral kernels, $K^{(+,+)}$satisfies the desired $L^{p}$-bounds, and so does $K^{(-,-)}$. To analyze $K^{(+,-)}$and $K^{(-,+)}$, we first note that

$$
c_{+}(k)=\frac{1}{2 i} \frac{1+\overline{m_{1}(0, k)}}{1+m_{1}(0, k)},
$$

so that by (21)

$$
\left|\partial_{k}^{j}\left[\mu(k) c_{+}(k) m(r, k) m\left(r^{\prime}, k\right)\right]\right| \lesssim k^{-j}, \quad j=0,1,2
$$

Hence,

$$
\left|K^{( \pm, \mp)}\left(r, r^{\prime}\right)\right| \lesssim\left(r+r^{\prime}\right)^{-1}
$$

Since this latter kernel is well known to be $L^{p}(0, \infty)$ bounded for $1<p<\infty$, we are done.

Next, we consider small energies.

Lemma 3. Let $\mu \in C^{3}(0, \infty)$ with $\left|\mu^{(\ell)}(k)\right| \leq k^{-\ell}$ for all $k>0$ and $\ell=0,1,2,3$. Assume further that $\mu(k)=0$ for all $k>1$. Then the kernel $K\left(r, r^{\prime}\right)$ of $\widetilde{\mathcal{M}}_{\mu}=$ $\tilde{\mathcal{F}}_{V}^{-1} \mu \tilde{\mathcal{F}}_{V}$ satisfies

$$
K=K_{1}+K_{2}+K_{3},
$$

where

$$
\begin{aligned}
K_{1}\left(r, r^{\prime}\right) & =\frac{1}{2} m(r, 0) m\left(r^{\prime}, 0\right) \int_{0}^{\infty} \cos \left(\left(r-r^{\prime}\right) k\right) \mu(k) d k \\
\left|K_{2}\left(r, r^{\prime}\right)\right| & \lesssim\left|r-r^{\prime}\right|^{-1}, \quad\left|\partial_{r} K_{2}\left(r, r^{\prime}\right)\right|+\left|\partial_{r^{\prime}} K_{2}\left(r, r^{\prime}\right)\right| \lesssim\left|r-r^{\prime}\right|^{-2}, \\
\left|K_{3}\left(r, r^{\prime}\right)\right| & \lesssim\left(r+r^{\prime}\right)^{-1},
\end{aligned}
$$

for all $r, r^{\prime} \in(0, \infty)$. All these kernels are bounded on $L^{p}(0, \infty)$ for $1<p<\infty$.

Proof. We will again rely on the decompositions (18) and (19). With $\psi$ our Littlewood-Paley function, we further write

$$
\begin{aligned}
K^{(+,+)}\left(r, r^{\prime}\right) & =\frac{1}{4} \int_{0}^{\infty} e^{i\left(r-r^{\prime}\right) k} \mu(k) m(r, 0) \overline{m\left(r^{\prime}, 0\right)} d k \\
& +\sum_{j<0} \frac{1}{4} \int_{0}^{\infty} e^{i\left(r-r^{\prime}\right) k} \psi\left(2^{-j} k\right) \mu(k) m\left(r, r^{\prime} ; k\right) d k \\
& =: K_{0}^{(+,+)}\left(r, r^{\prime}\right)+\sum_{j<0} K_{j}^{(+,+)}\left(r, r^{\prime}\right)
\end{aligned}
$$

${ }^{5}$ If $f(0,0)=0$, then $K_{1}=0$. 
with

$m\left(r, r^{\prime} ; k\right)=m(r, k) \overline{m\left(r^{\prime}, k\right)}-m(r, 0) \overline{m\left(r^{\prime}, 0\right)}=m(r, k) \overline{m\left(r^{\prime}, k\right)}-m(r, 0) m\left(r^{\prime}, 0\right)$.

Since $m(\cdot, k) \in L^{\infty}(0, \infty)$, the first integral $K_{0}^{(+,+)}$on the right-hand side of (22) is bounded $L^{p}(0, \infty) \rightarrow L^{p}(0, \infty)$ for $1<p<\infty$. Here we are again using the fact that

$$
\int_{0}^{\infty} e^{i\left(r-r^{\prime}\right) k} \mu(k) d k
$$

is a singular integral kernel. To obtain $K_{1}\left(r, r^{\prime}\right)$, define

$$
K_{1}\left(r, r^{\prime}\right)=K_{0}^{(+,+)}\left(r, r^{\prime}\right)+K_{0}^{(-,-)}\left(r, r^{\prime}\right) .
$$

We now claim that

$$
\left|\partial_{r} K_{j}^{(+,+)}\left(r, r^{\prime}\right)\right| \lesssim \min \left(2^{2 j},\left|r-r^{\prime}\right|^{-3} 2^{-j}\right) \quad \forall j<0, \forall r, r^{\prime}>0,
$$

and symmetrically with $\partial_{r^{\prime}}$. This in turn implies that

$$
\tilde{K}^{(+,+)}\left(r, r^{\prime}\right):=\sum_{j<0} K_{j}^{(+,+)}\left(r, r^{\prime}\right)
$$

satisfies

$$
\left|\partial_{r} \tilde{K}^{(+,+)}\left(r, r^{\prime}\right)\right| \lesssim\left|r-r^{\prime}\right|^{-2},
$$

from which we obtain the Hörmander condition

$$
\sup _{r_{1}, r_{2}>0} \int_{\left[\left|r^{\prime}-r_{1}\right|>2\left|r_{1}-r_{2}\right|\right]}\left|\tilde{K}^{(+,+)}\left(r_{1}, r^{\prime}\right)-\tilde{K}^{(+,+)}\left(r_{2}, r^{\prime}\right)\right| d r^{\prime}<\infty .
$$

To prove (23), let $h(a)=\frac{e^{2 i a}-1}{2 i a}$. This function satisfies $\left|h^{(\ell)}(a)\right| \lesssim\langle a\rangle^{-\ell-1}$. Then (20) is the same as

$$
m(r, k)=1+\int_{r}^{\infty} h\left(k\left(r^{\prime}-r\right)\right)\left(r^{\prime}-r\right) V\left(r^{\prime}\right) m\left(r^{\prime}, k\right) d r^{\prime}
$$

and thus

$$
\begin{aligned}
\partial_{k} m(r, k) & =\int_{r}^{\infty} h^{\prime}\left(k\left(r^{\prime}-r\right)\right)\left(r^{\prime}-r\right)^{2} V\left(r^{\prime}\right) m\left(r^{\prime}, k\right) d r^{\prime} \\
& +\int_{r}^{\infty} h\left(k\left(r^{\prime}-r\right)\right)\left(r^{\prime}-r\right) V\left(r^{\prime}\right) \partial_{k} m\left(r^{\prime}, k\right) d r^{\prime}
\end{aligned}
$$


as well as

$$
\begin{aligned}
\partial_{r} \partial_{k} m(r, k) & =-k \int_{r}^{\infty} h^{\prime \prime}\left(k\left(r^{\prime}-r\right)\right)\left(r^{\prime}-r\right)^{2} V\left(r^{\prime}\right) m\left(r^{\prime}, k\right) d r^{\prime} \\
& -2 \int_{r}^{\infty} h^{\prime}\left(k\left(r^{\prime}-r\right)\right)\left(r^{\prime}-r\right) V\left(r^{\prime}\right) m\left(r^{\prime}, k\right) d r^{\prime} \\
& -\int_{r}^{\infty} e^{2 i k\left(r^{\prime}-r\right)} V\left(r^{\prime}\right) \partial_{k} m\left(r^{\prime}, k\right) d r^{\prime}
\end{aligned}
$$

From these identities we obtain the bounds

$$
\sup _{0<k<1} \sup _{r>0}\left[\left|\partial_{k} m(r, k)\right|+\left|\partial_{r} \partial_{k} m(r, k)\right|\right]<\infty
$$

and therefore alsd 6

$$
\sup _{r>0}\left(\left|\partial_{r}(m(r, k)-m(r, 0))\right|+|m(r, 0)-m(r, k)|\right) \lesssim k,
$$

which finally implies

$$
\sup _{r, r^{\prime}>0}\left(\left|m\left(r, r^{\prime} ; k\right)\right|+\left|\partial_{r} m\left(r, r^{\prime} ; k\right)\right|\right) \lesssim k .
$$

These bounds give us one-half of the claim (23). Indeed,

$$
\left|\partial_{r} K_{j}^{(+,+)}\left(r, r^{\prime}\right)\right| \lesssim \int_{0}^{\infty}\left|\psi\left(2^{-j} k\right)\right| k d k \lesssim 2^{2 j}
$$

To obtain the other half, we estimate

$$
\begin{aligned}
\left|r-r^{\prime}\right|^{3}\left|\partial_{r} K_{j}^{(+,+)}\left(r, r^{\prime}\right)\right| & \lesssim \int_{0}^{\infty}\left|\partial_{k}^{3}\left[k \psi\left(2^{-j} k\right) \mu(k) m\left(r, r^{\prime} ; k\right)\right]\right| d k \\
& +\int_{0}^{\infty}\left|\partial_{k}^{3}\left[\psi\left(2^{-j} k\right) \mu(k) \partial_{r} m\left(r, r^{\prime} ; k\right)\right]\right| d k .
\end{aligned}
$$

These estimates imply the remaining half of (23), viz.

$$
\left|r-r^{\prime}\right|^{3}\left|\partial_{r} K_{j}^{(+,+)}\left(r, r^{\prime}\right)\right| \lesssim 2^{-j}
$$

provided we can prove that 7

$$
\sup _{r, r^{\prime}>0}\left[\left|\partial_{k}^{\ell} m\left(r, r^{\prime} ; k\right)\right|+\left|\partial_{k}^{\ell} \partial_{r} m\left(r, r^{\prime} ; k\right)\right|\right] \lesssim k^{1-\ell} \quad \forall \ell \geq 0 .
$$

\section{Writing}

$$
\begin{aligned}
m\left(r, r^{\prime} ; k\right) & =(m(r, k)-m(r, 0)) m\left(r^{\prime}, k\right)+m(r, 0)\left(m\left(r^{\prime}, k\right)-m\left(r^{\prime}, 0\right)\right), \\
\partial_{r} m\left(r, r^{\prime} ; k\right) & =\left(\partial_{r} m(r, k)-\partial_{r} m(r, 0)\right) m\left(r^{\prime}, k\right)+\partial_{r} m(r, 0)\left(m\left(r^{\prime}, k\right)-m\left(r^{\prime}, 0\right)\right),
\end{aligned}
$$

\footnotetext{
${ }^{6}$ Automatically in this proof $0<k<1$.

${ }^{7}$ We only need this for $\ell \leq 3$, but it holds for all $\ell$.
} 
it is easy to see that (28) follows from (25) and (26). For example,

$$
\begin{aligned}
\partial_{k}^{2} m(r, k)= & k^{-1} \int_{r}^{\infty} h^{\prime \prime}\left(k\left(r^{\prime}-r\right)\right) k\left(r^{\prime}-r\right)\left(r^{\prime}-r\right)^{2} V\left(r^{\prime}\right) m\left(r^{\prime}, k\right) d r^{\prime} \\
& +2 \int_{r}^{\infty} h^{\prime}\left(k\left(r^{\prime}-r\right)\right)\left(r^{\prime}-r\right)^{2} V\left(r^{\prime}\right) \partial_{k} m\left(r^{\prime}, k\right) d r^{\prime} \\
& +\int_{r}^{\infty} h\left(k\left(r^{\prime}-r\right)\right)\left(r^{\prime}-r\right) V\left(r^{\prime}\right) \partial_{k}^{2} m\left(r^{\prime}, k\right) d r^{\prime}
\end{aligned}
$$

proves that $\left|\partial_{k}^{2} m(r, k)\right| \lesssim k^{-1}$ and inductively, $\left|\partial_{k}^{\ell} m(r, k)\right| \lesssim k^{1-\ell}$ for all $\ell \geq 2$. Similarly,

$$
\begin{aligned}
\partial_{r} \partial_{k}^{2} m(r, k)= & -\int_{r}^{\infty} h^{\prime \prime}\left(k\left(r^{\prime}-r\right)\right)\left(r^{\prime}-r\right)^{2} V\left(r^{\prime}\right) m\left(r^{\prime}, k\right) d r^{\prime} \\
& -\int_{r}^{\infty} h^{\prime \prime \prime}\left(k\left(r^{\prime}-r\right)\right) k\left(r^{\prime}-r\right)\left(r^{\prime}-r\right)^{2} V\left(r^{\prime}\right) m\left(r^{\prime}, k\right) d r^{\prime} \\
& -k \int_{r}^{\infty} h^{\prime \prime}\left(k\left(r^{\prime}-r\right)\right)\left(r^{\prime}-r\right)^{2} V\left(r^{\prime}\right) \partial_{k} m\left(r^{\prime}, k\right) d r^{\prime} \\
& -2 \int_{r}^{\infty} h^{\prime \prime}\left(k\left(r^{\prime}-r\right)\right)\left(r^{\prime}-r\right)^{2} V\left(r^{\prime}\right) \partial_{k} m\left(r^{\prime}, k\right) d r^{\prime} \\
& -2 \int_{r}^{\infty} h^{\prime}\left(k\left(r^{\prime}-r\right)\right)\left(r^{\prime}-r\right) V\left(r^{\prime}\right) \partial_{k}^{2} m\left(r^{\prime}, k\right) d r^{\prime} \\
& -2 i \int_{r}^{\infty} e^{2 i k\left(r^{\prime}-r\right)}\left(r^{\prime}-r\right) V\left(r^{\prime}\right) \partial_{k} m\left(r^{\prime}, k\right) d r^{\prime} \\
& +V(r) \partial_{k} m(r, k)
\end{aligned}
$$

implies that $\left|\partial_{r} \partial_{k}^{2} m(r, k)\right| \lesssim k^{-1}$, and analogously for the higher derivatives.

To summarize,

$$
K_{2}\left(r, r^{\prime}\right)=\tilde{K}^{(+,+)}\left(r, r^{\prime}\right)+\tilde{K}^{(-,-)}\left(r, r^{\prime}\right)
$$

satisfies

$$
\left|\partial_{r} K_{2}\left(r, r^{\prime}\right)\right|+\left|\partial_{r^{\prime}} K_{2}\left(r, r^{\prime}\right)\right| \lesssim\left|r-r^{\prime}\right|^{-2}
$$

and therefore a Hörmander condition. It is easier and also implicit in the preceding to check that

$$
\left|K_{2}\left(r, r^{\prime}\right) \lesssim\right| r-\left.r^{\prime}\right|^{-1}
$$

We also need to estimate the kernel

$$
K_{3}\left(r, r^{\prime}\right):=K^{(+,-)}\left(r, r^{\prime}\right)+K^{(-,+)}\left(r, r^{\prime}\right)
$$

from (19). For those we remark that the bounds

$$
\left|\partial_{k}^{\ell}\left[\mu(k) c_{+}(k) m(r, k) m\left(r^{\prime}, k\right)\right]\right| \lesssim k^{-\ell}, \quad \ell=0,1,2,
$$


which follow easily from our preceding work, imply

$$
\left|K^{(+,-)}\left(r, r^{\prime}\right)\right|+\left|K^{(-,+)}\left(r, r^{\prime}\right)\right| \lesssim\left(r+r^{\prime}\right)^{-1} .
$$

However, this latter kernel $\left(r+r^{\prime}\right)^{-1}$ is $L^{p}(0, \infty)$ bounded.

We still need to establish the $L^{p}$ boundedness of $\tilde{K}^{(+,+)}\left(r, r^{\prime}\right)+\tilde{K}^{(-,-)}\left(r, r^{\prime}\right)$. Now suppose that this sum is an $L^{2}$ bounded operator. Since we have a Hörmander condition, the Calderon-Zygmund theorem (see Stein Ste2], page 19, Theorem 3) will then guarantee that it is bounded on $L^{p}$ with $1<p \leq 2$, and by taking adjoints, this will then also hold for $2 \leq p<\infty$. Hence it suffices to check the case $p=2$. First, $\tilde{\mathcal{M}}_{\mu}$ with kernel $K\left(r, r^{\prime}\right)$ is $L^{2}$ bounded by the spectral theorem. Second,

$$
\begin{aligned}
\tilde{K}^{(+,+)}\left(r, r^{\prime}\right)+\tilde{K}^{(-,-)}\left(r, r^{\prime}\right) & =K\left(r, r^{\prime}\right)-K_{0}^{(+,+)}\left(r, r^{\prime}\right)-K_{0}^{(-,-)}\left(r, r^{\prime}\right) \\
& -K^{(+,-)}\left(r, r^{\prime}\right)-K^{(-,+)}\left(r, r^{\prime}\right)
\end{aligned}
$$

is therefore also $L^{2}$ bounded. The lemma is proved.

These two lemmas establish $L^{p}(0, \infty)$ boundedness of $\widetilde{\mathcal{M}}_{\mu}$ for any Mikhlin multiplier $\mu$ on the half-line. However, we are really after the $L^{p}\left(\mathbb{R}^{3}\right)$-boundedness of $\mathcal{M}_{\mu}$. In view of (11), this is equivalent to a certain weighted $L^{p}$ boundedness of $\widetilde{\mathcal{M}}_{\mu}$. The theory of $A_{p}$ weights will give us what we need, as we shall now see. It is in order to apply the theory of $A_{p}$ weights that we have expressed the kernels explicitly as in the previous two lemmas.

Proof of Proposition 1. First, observe that for radial functions $f, g$ in $\mathbb{R}^{3}$,

$$
\left\langle\mathcal{M}_{\mu} f, g\right\rangle=\left\langle\widetilde{\mathcal{M}}_{\mu} \tilde{f}, \tilde{g}\right\rangle
$$

and thus

$$
\begin{aligned}
\left\|\mathcal{M}_{\mu} f\right\|_{p} & =\sup _{\|g\|_{p^{\prime}}=1}\left|\left\langle\widetilde{\mathcal{M}}_{\mu} \tilde{f}, \tilde{g}\right\rangle\right| \\
& =\sup _{\left\|r^{\frac{2}{p^{\prime}}-1} \tilde{g}\right\|_{L^{p^{\prime}}(0, \infty)}=1}\left|\left\langle r^{1-\frac{2}{p^{\prime}}} \widetilde{\mathcal{M}}_{\mu} \tilde{f}, r^{\frac{2}{p^{\prime}}-1} \tilde{g}\right\rangle\right| \\
& =\left\|r^{\frac{2}{p}-1} \widetilde{\mathcal{M}}_{\mu} \tilde{f}\right\|_{L^{p}(0, \infty)} .
\end{aligned}
$$

Hence, we need to prove that

$$
\left\|r^{\frac{2}{p}-1} \widetilde{\mathcal{M}}_{\mu} \tilde{f}\right\|_{p} \lesssim\left\|r^{\frac{2}{p}-1} \tilde{f}\right\|_{p}
$$

on the half-line. We now claim that $\omega(r)=r^{2-p}$ is an $A_{p}$-weight for all $3 / 2<p<3$. Recall that this means that

$$
\sup _{b>a \geq 0} f_{a}^{b} \omega(x) d x\left(\int_{a}^{b} \omega^{-\frac{p^{\prime}}{p}}(x) d x\right)^{\frac{p}{p^{\prime}}}<\infty,
$$

where $f$ is the averaged integral. To check this for our weight $\omega(r)=r^{2-p}$, we first remark that due to $3 / 2<p<3$, we have $1>(2-p) p^{\prime} / p$ and $2-p>-1$. Hence, if $b \gg a$, then the left-hand side of (29) is

$$
\sup _{b>0} \lesssim b^{2-p}\left(b^{-(2-p) p^{\prime} / p}\right)^{p / p^{\prime}} \lesssim 1
$$

If $b \lesssim a$, then one applies the mean-value theorem to reach a similar conclusion. 
The main property of $A_{p}$ weights is that the Hardy-Littlewood maximal operator, as well as Calderon-Zygmund operators, are bounded on $L^{p}(\omega)$. Since one can check this manually for $\omega(r)=r^{2-p}$ and the kernel $\left(r+r^{\prime}\right)^{-1}$, the proposition now follows from Lemmas 2 and 3 ,

It is now standard to pass from Proposition 1 to the Littlewood-Paley theorem. Let $\sum_{j} \psi\left(2^{-j} k\right)=1, k \neq 0$, be the usual Littlewood-Paley decomposition of unity.

Corollary 4. Let $H$ be as in Proposition 1 and define

$$
S_{H} f=\left(\sum_{j}\left|\psi\left(2^{-j} \sqrt{H}\right) f\right|^{2}\right)^{\frac{1}{2}}
$$

to be the square function relative to $H$. Then

$$
C(V, p)^{-1}\|f\|_{p} \leq\left\|S_{H} f\right\|_{p} \leq C(V, p)\|f\|_{p}
$$

for all radial $f \in L_{c}^{2}\left(\mathbb{R}^{3}\right) \cap L^{p}\left(\mathbb{R}^{3}\right)$ with $3 / 2<p<3$, where $L_{c}^{2}\left(\mathbb{R}^{3}\right)$ is the continuous subspace of $H$.

Proof. The upper bound follows from (13) by means of the usual randomization and Khinchin inequality method, whereas the lower bound in (30) then follows from the upper bound via duality. We skip the details.

\section{Concluding Remarks}

- It would be interesting to extend the results here to the case of nonradial functions, as well as nonradial potentials. In this case it is most likely necessary to estimate the solutions of the Lippman-Schwinger equation directly. Here we choose to work in the radial setting, since the ensuing reduction to the half-line allows us to work with a Volterra integral equation, rather than the more difficult Fredholm integral equation (as in Lippman-Schwinger). The Volterra equation of course is the one governing the Jost solutions. At any rate, in the general case it will be necessary to isolate the singularity of the perturbed resolvent at energy zero as in Jensen and Kato JenKat, or Jensen and Nenciu JenNen]. See also [ErdSch, where the Jensen-Nenciu method is carried out in $\mathbb{R}^{3}$.

- As mentioned before, Strichartz estimates for the wave equation with a potential remain open for potentials that lead to singular resolvents at zero energy. We are referring here to Strichartz estimates on the regular part of the evolution (see $\mathcal{S}_{a}$ in (8) ) and wish to obtain the full range of exponents as in the free case. This seems plausible, because of the dispersive estimate (10) on the one hand (albeit with a nonhomogeneous norm on the right-hand side), and the energy bound

$$
\sup _{t \geq 0}\left\|\sqrt{H_{a}} P_{c} \mathcal{S}_{a}(t) P_{c} f\right\|_{2} \lesssim\|f\|_{2}
$$

on the other hand.

- Finally, we would like to point out that in the radial case, as considered here, Strichartz estimates for the free wave equation do not require Littlewood-Paley theory at all. Indeed, they can be obtained by interpolation between the "forbidden endpoint" $\left(L^{\infty}\left(\mathbb{R}^{3}\right)\right.$ in space) and the energy bound; see Sogge's book Sog. It is conceivable that this approach also yields something for the case of the wave equation with a radial potential. 


\section{ACKNOWLEDGEMENTS}

The author wishes to thank Sigmund Selberg from NTNU in Trondheim, Norway, for his hospitality and for its support the Research Council of Norway, through the research project "Partial Differential Equations and Harmonic Analysis". Also, he wishes to thank Fritz Gesztesy for many helpful discussions, and Burak Erdogan and Marius Beceanu for comments on a preliminary version of this paper.

\section{REFERENCES}

[Agm] Agmon, S. Spectral properties of Schrödinger operators and scattering theory. Ann. Scuola Norm. Sup. Pisa Cl. Sci. (4) 2 (1975), no. 2, 151-218. MR0397194 (53:1053)

[Aub] Aubin, T. Nonlinear analysis on manifolds. Monge-Ampère equations. Grundlehren der Mathematischen Wissenschaften, 252. Springer-Verlag, New York, 1982. MR0681859 (85j:58002)

[ErdSch] Erdoğan, M. B., Schlag, W. Dispersive estimates for Schrödinger operators in the presence of a resonance and/or an eigenvalue at zero energy in dimension three: I, Dynamics of PDE, vol. 1, no. 4 (2004), 359-379. MR 2127577

[GesZin] Gesztesy, F., Zinchenko, M. On spectral theory of Schrödinger operators with strongly singular potentials. preprint 2005.

[JenKat] Jensen, A., Kato, T. Spectral properties of Schrödinger operators and time-decay of the wave functions. Duke Math. J. 46 (1979), no. 3, 583-611. MR0544248|(81b:35079)

[JenNak1] Jensen, A., Nakamura, S. $L^{p}$ and Besov estimates for Schrödinger Operators. Advanced Studies in Pure Math. 23, Spectral and Scattering Theory and Applications (1994), 187-209.

[JenNak2] Jensen, A., Nakamura, S. L ${ }^{p}$-mapping properties of functions of Schrödinger operators and their applications to scattering theory. J. Math. Soc. Japan 47 (1995), no. 2, 253273. MR:1317282 (95m:47087)

[JenNen] Jensen, A., Nenciu, G. A unified approach to resolvent expansions at thresholds. Rev. Math. Phys. 13 (2001), no. 6, 717-754. MR1841744 (2002e:81031)

[KriSch] Krieger, J., Schlag, W. On the focusing critical semi-linear wave equation, preprint 2005.

[Sog] Sogge, C. Lectures on nonlinear wave equations. Monographs in Analysis, II. International Press, Boston, MA, 1995. MR.1715192 (2000g:35153)

[Ste1] Stein, E. M. Topics in harmonic analysis related to the Littlewood-Paley theory. Annals of Mathematics Studies, No. 63, Princeton University Press, Princeton, N.J., University of Tokyo Press, Tokyo, 1970. MR0252961 (40:6176)

[Ste2] Stein, E. Harmonic analysis, Princeton University Press, Princeton, 2004.

[Tal] Talenti, G. Best constant in Sobolev inequality. Ann. Mat. Pura Appl. (4) 110 (1976), 353-372. MR0463908 (57:3846)

[Yaj] Yajima, K. The $W^{k, p}$-continuity of wave operators for Schrödinger operators. J. Math. Soc. Japan 47 (1995), no. 3, 551-581. MR1331331 (97f:47049)

[Yaj2] Yajima, K. Dispersive estimate for Schrödinger equations with threshold resonance and eigenvalue, preprint 2004, to appear in Comm. Math. Phys.

Department of Mathematics, University of Chicago, 5734 South University Ave., Chicago, Illinois 60637

E-mail address: schlag@math.uchicago.edu 